\section{Silk Genes Support the Single Origin of Orb Webs}

\author{
Jessica E. Garb, ${ }^{*}$ Teresa DiMauro, Victoria Vo, Cheryl Y. Hayashi
}

$\mathrm{T}$ he spider orb web is an impressive example of animal architecture. This silken net consists of a frame and supporting radii overlaid with a sticky capture spiral. Because an orb web must absorb the enormous kinetic impact of flying prey, silks composing the web have exceptional mechanical properties (1). Orb weaving is characteristic of species in two lineages: Araneoidea and Deinopoidea (Fig. 1A). Araneoid and deinopoid orb weavers use nearly identical behavioral sequences and
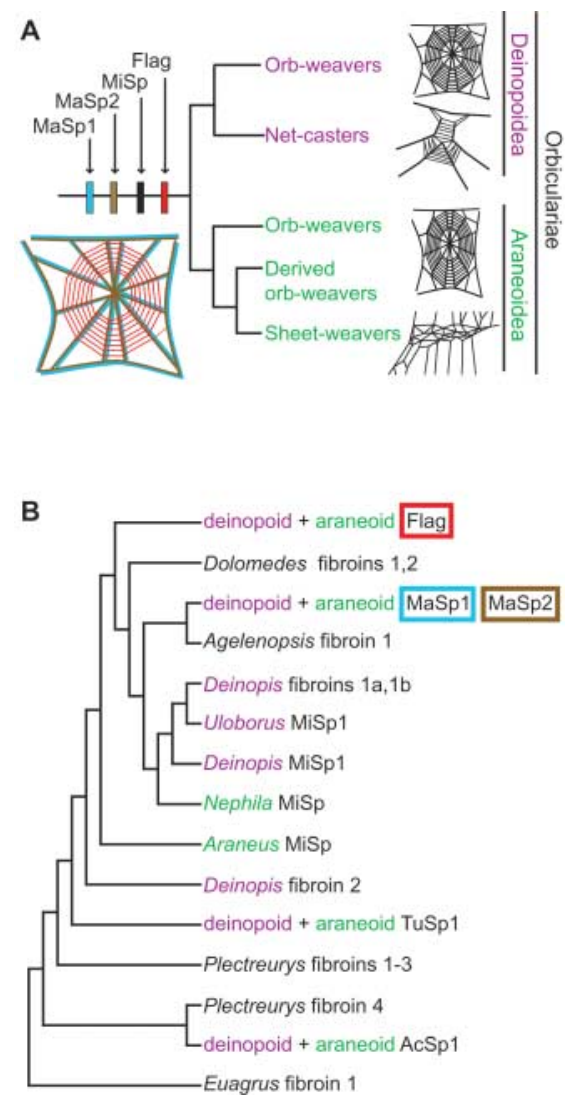

Fig. 1. Relationships of orb-weaving spiders and spidroins. (A) Deinopoid (purple) and araneoid (green) lineages (2), depicting inferred ancestral web and spidroins. Orb-web frame and radii composed of MaSp1 (blue) and MaSp2 (brown) are shown along with temporary spiral with MiSp (black) and capture spiral with Flag (red). (B) Summarized phylogeny of spidroin family members. The full tree is shown in fig. S2. spinning apparatuses to produce architecturally similar webs. However, there are notable differences between the adhesive mechanisms of capture spirals spun by araneoids (aqueous glue) and deinopoids (dry fibrils) (2). Thus, the two types of orb webs were widely considered a dramatic example of convergent evolution (3).

Spider silks are composed of proteins (spidroins) synthesized in specialized abdominal glands. Three types of glands produce the fibers used in orb-web construction: (i) major ampullates for frame and radial fibers, (ii) minor ampullates for temporary spiral scaffolding, and (iii) flagelliforms for capture spiral axial fibers (4). Whereas silk cDNAs were described from these glands in araneoids, none are known from deinopoids. If Araneoidea and Deinopoidea are sister taxa (Orbiculariae), then the simplest explanation for similarities between their orb webs is that they were acquired from a common orb-weaving ancestor (2). Thus, deinopoids are expected to have counterparts to araneoid silk proteins used in orb-web construction. To test this hypothesis, we characterized silk gland cDNAs from representatives of both deinopoid families: a net-casting spider, Deinopis spinosa (Deinopidae), and a feather-legged orb weaver, Uloborus diversus (Uloboridae) (5).

Among the deinopoid cDNAs, we identified the following orthologs of araneoid silks used in orb webs: (i) MaSp1 and MaSp2, major ampullate proteins of the frame and radii, (ii) MiSp, minor ampullate scaffolding protein, and (iii) Flag, flagelliform protein of capture spirals (4) (fig. S1). Given the diversification of silk glands and fiber types that accompanied spider evolution (6), the distribution of certain spidroins should uniquely define particular clades. Spidroins have been reported from six nonorbicularian species (4), but MiSp and Flag are exclusively known from araneoids, and major ampullate cDNAs that encode MaSp1-like spidroins but not MaSp2 are known from nonorbicularians. Because minor and major ampullate gland spigots are broadly distributed in nonorbicularians (6), the use of MiSp and MaSp1 by araneoids and deinopoids is interpreted as plesiomorphic. However, araneoids and deinopoids are united by their shared use of MaSp2. Moreover, araneoids and deinopoids are the only spiders that have flagelliform-like spigots and make capture spirals with silk from these spigots (2). The discovery of Flag and $\mathrm{MaSp} 2$ in deinopoids consequently identifies these spidroins as molecular synapomorphies (shared derived traits) supporting orbicularian monophyly.

Phylogenetic analyses of spidroin C-termini substantiate close relationships between deinopoid and araneoid spidroins used in orb webs (Fig. 1B). The analyses do not consistently recover a grouping of MiSp-like sequences, but the resulting trees do contain clades of the following: (i) deinopoid and araneoid Flag, (ii) deinopoid and araneoid MaSp1 and MaSp2, (iii) deinopoid and araneoid AcSp1 (prey-wrapping silk protein), and (iv) deinopoid and araneoid TuSp1 (eggcase silk protein). The collective combination of Flag, MiSp, MaSp1, and MaSp2 in araneoid and deinopoid spiders implies that the orbicularian ancestor was equipped with the molecular elements necessary for orb-web construction. Based on fossil evidence, this ancestor minimally dates from the Lower Cretaceous, 136 million years ago (7). Accordingly, the birth of Flag, MiSp, MaSp1, and MaSp2 must have occurred by this time. Since their ancient origins, these silks have diversified during the subsequent radiation of Orbiculariae ( $>10,000$ species) (2), thus greatly expanding the designs available for the production of high-performance biomimetic materials.

References and Notes

1. F. Vollrath, D. P. Knight, Nature 410, 541 (2001).

2. C. E. Griswold, J. A. Coddington, G. Hormiga, N. Scharff, Zool. J. Linn. Soc. 123, 1 (1998).

3. W. A. Shear, Ed., Spiders: Webs, Behavior, and Evolution. (Stanford Univ. Press, Stanford, CA, 1986).

4. J. Gatesy, C. Hayashi, D. Motriuk, J. Woods, R. Lewis, Science 291, 2603 (2001).

5. Materials and methods are available as supporting material on Science Online.

6. N. Platnick, ]. Coddington, R. Forster, C. Griswold, Am. Mus. Novit. 3016, 1 (1991).

7. P. A. Selden, Nature 340, 711 (1989).

8. N. Nguyen, P. Paquin, and M. Stowe provided assistance. N. Ayoub, T. Blackledge, J. Gatesy, M. McGowen, D. Reznick, and A. Suarez commented on drafts. Work was supported by NSF grant no. DEB-0236020 and by the Army Research Office grant no. DAAD19-02-1-0358. Sequences were deposited in GenBank with accession codes DQ399323 to DQ399335.

Supporting Online Material

www.sciencemag.org/cgi/content/full/312/5781/1762/DC1 Materials and Methods

Figs. $\mathrm{S} 1$ and $\mathrm{S} 2$

References

27 March 2006; accepted 28 April 2006 10.1126/science. 1127946

Department of Biology, University of California, Riverside, CA 92521, USA.

${ }^{*}$ To whom correspondence should be addressed. E-mail: jessica.garb@ucr.edu 


\section{Science \\ DIAAAS}

\section{Supporting Online Material for}

\section{Silk Genes Support the Single Origin of Orb Webs}

Jessica E. Garb,* Teresa DiMauro, Victoria Vo, Cheryl Y. Hayashi

*To whom correspondence should be addressed. E-mail: jessica.garb@ucr.edu

Published 23 June 2006, Science 312, 1762 (2006)

DOI: $10.1126 /$ science.1127946

\section{This PDF file includes:}

Materials and Methods

Figs. S1 and S2

References 


\section{Supporting Online Material for}

Silk genes support the single origin of orb-webs

Jessica E. Garb*, Teresa DiMauro, Victoria Vo, and Cheryl Y. Hayashi

*To whom correspondence should be addressed. E-mail: jessica.garb@ucr.edu

\section{This supporting material includes:}

Materials and Methods

Figs. S1 and S2

References 


\section{Materials and Methods}

Silk gland tissue was dissected from Uloborus diversus (Riverside, CA) and Deinopis spinosa (Gainesville, FL) and flash-frozen. cDNA libraries were constructed from each species using methods in $S 1$. Libraries were probed with these oligonucleotides (also those in 51 ): AAGCAGCATTCAAGACAGC, CCAGTAAATCCTGTCAAGCC, CTTGTTGCTGGGAAGAAAG, ATCCACCTAGATTTCCTTG, TGTTGAWATGYTGGCGGTCC, CCWCCWGGWCCNNNWCCWCCWGGWCC, CCWGGWCCTTGTTGWCCWGGWCC, AGTTGTTGTAGTGGTTGTCGT, TTGCTTGCRTTGASAACWCC, GARGGKCCAGARGGWCC, AACTGGTCCGTAACCTCTG, AGGGAAGAAACAGCCGAAG, ACTAACCGATGAGGAGAGG, GAGTCCTCCTCTGTAGTA, CCTCGTCCTCCAAGTGCTATACT, and GAGAGGGCAGATGCATCAGCGGAA. In addition to the probe-positives, subsamples of clones with large inserts were sequenced from each library using methods described in $S 1$. From hundreds of sequenced clones, we identified clusters of distinct silk cDNAs. The longest insert from each group was entirely sequenced (data summarized in Fig. S1). A longer sequence of Uloborus MaSp2 was obtained by PCR amplifying genomic DNA (primers

GCAGCCGCTGCAGCAGCAGC and AAACATCAACTGGTCCGAC) and sequencing cloned PCR fragments.

We used established criteria (S2) to assign silks to MaSp1, MaSp2, MiSp and Flag ortholog groups. Note that the deinopoid Flag spidroin contains $\sim 40$ tandem GPQX $\mathrm{n}_{\mathrm{n}}$ and GPGX $_{\mathrm{n}}$ subunits. Top BLASTX hits to Deinopis Flag in the correct reading frame were other Flag spidroins. Algorithmic predictions of secondary structure of Deinopis Flag using the Chou-Fasman and Robson-Garnier methods indicate it is almost entirely composed of consecutive $\beta$-turns, similar to araneoid Flag. We also identified Uloborus AcSp1 based on sequence similarity to the published AcSp1. Spidroin nomenclature follows the practice where the first two letters of a gene (and inferred protein) indicate the gland where initially isolated $(\mathrm{Ma}=$ major ampullate, $\mathrm{Mi}=$ minor ampullate, $\mathrm{Tu}=$ tubuliform, $\mathrm{Ac}=$ aciniform; excepting Flag= flagelliform), preceding "Sp" for spidroin. Spidroins not assignable to an ortholog group are labeled "fibroin \#" (numbers indicate different paralogs) (S2). Examples reported here are Deinopis fibroin 1a, fibroin 1b, and fibroin 2. Another discovered cDNA, SGP-1, has the repetitive organization of spidroins but lacks a spidroin-like C-terminus. Thus, SGP-1 may be a spidroin family member that lost its spidroin-like C-terminus or is outside the spidroin family. SGP-1 appears to be a silk protein, as its iterated repeats are consistent with fiber formation and its transcripts represented the most abundant cDNA in Deinopis libraries. Deinopis fibroin 1a, fibroin $1 \mathrm{~b}$, fibroin 2, and SGP-1 may represent constituents of a previously uncharacterized silk, such as piriform or cribellate silk.

Relationships of spidroins reported here (Genbank accession: DQ399323-DQ399335) were analyzed with published spidroins. From Araneoidea, we included Nephila clavipes (N.c.), N.c. MaSp1 (U20329), N.c. MaSp2 (M92913), N.c. MiSp1 (AF027735), N.c. MiSp 
(AF027736), N.c. TuSpl (AY855102), N.c. Flag (AF027973); Araneus diadematus (A.d.), A.d. MiSp (ADF1; U47853), A.d. MaSp1 (ADF2; U47854), A.d. MaSp2a (ADF3; U47855), A.d. MaSp2b (ADF4; U47856); Argiope trifasciata (A.t.), A.t. MaSp1 (AF350266), A.t. MaSp2 (AF350267), A.t. AcSp1 (AY426339), A.t. Flag (AF350264); Argiope argentata (A.ar.), A.ar. TuSpl (AY953071); Latrodectus hesperus (L.h.), L.h. MaSpl (AY953074), L.h. MaSp2 (AY953075), L.h. TuSpl (AY953070). Included nonaraneoid spidroin sequences were as follows: from Uloborus diversus (U.d.), U.d. TuSp1 (AY953072); Deinopis spinosa (D.s.), D.s. TuSp1 (AY953073); Agelenopsis aperta (A.ap.), A.ap. fibroin 1 (AY566305); Dolomedes tenebrosus (D.t.), D.t. fibroin 1 (AF350269), D.t. fibroin 2 (AF350270); Euagrus chisoseus (E.c.), E.c. fibroin 1 (AF350271); Plectreurys tristis (P.t.), P.t. fibroin 1 - P.t. fibroin 4 (AF350281AF350284). Tai et al. (S3) reported MaSp1 from a deinopoid, Octonoba varians, and four additional species. These sequences, generated by PCR with heterologous primers designed from Nephila clavipes MaSp1, are nearly identical. Analyses of sequences from $S 3$ reveal that they group tightly together (100\% bootstrap support) and were sister to $N$. clavipes MaSpl (78\% bootstrap). Thus, we did not include the sequences in $S 3$ in our analyses because they apparently are PCR contaminants from N. pilipes MaSpl.

Spidroin C-terminal sequences were aligned using previously described methods $(S 1)$. The amino acid matrix contained 111 characters (105 variable). Parsimony analyses conducted in PAUP* 4b (S4), were unordered or employed a step matrix, specifying the minimum nucleotide substitutions required to convert one amino acid to another treating the two serines as different states. Gaps were treated as missing or presence/absence characters. Searches included 1000 random-taxon-additions, with TBR branch swapping. Support was computed from 1000 bootstrap (BS) replicates and decay indices (DI). Bayesian searches were implemented with Mr.Bayes 3.1.2 (S5), with a JTT amino acid model and gamma parameter of among-site rate variation. Runs were executed for 6 X $10^{6}$ generations, sampling every 1000 generations. Clade posterior probabilities were determined from trees retained after burn-in. Parsimony tree searches employing the step-matrix and recoded gaps produced one most parsimonious tree (1698 steps) (Fig. S2). Similar trees were derived using other methods, but differed in the arrangement of MaSp1-like, MaSp2-like and MiSp-like sequences (e.g., MiSp sequences form a clade in parsimony searches treating characters as unordered states but not in some of the other analyses).

In all trees, MaSp1-like sequences and MaSp2-like sequences never formed mutually exclusive clades. Instead, certain sequences containing MaSp1-like repeat modules consistently appear more closely related to sequences containing MaSp2-like repeats. Specifically, there is strong support for groupings of MaSp1 and MaSp2 C-termini from Uloborus $(\mathrm{BS}=90, \mathrm{DI}=6)$ and from Latrodectus $(\mathrm{BS}=98, \mathrm{DI}=10)$. This pattern suggests that non-vertical evolutionary processes may be operating on the MaSp1 and MaSp2 loci. For example, gene conversion events following non-reciprocal recombination between MaSp1 and MaSp2 could result in recombinant alleles in which the C-terminus of one locus is exchanged with the $\mathrm{C}$-terminus of the second. Aberrant recombination events 
between MaSp1 and MaSp2 loci may be facilitated by base pairing between their polyalanine coding sequences, especially given similar codon usage biases (S6). Another possible explanation for the grouping of MaSp1 and MaSp2 C-termini, which may also involve gene conversion, concerns the possibility of selection for similar C-terminal sequences in MaSp1 and MaSp2. Because MaSp1 and MaSp2 molecules aggregate with each other in the major ampullate glands and co-polymerize to form silk fibers, they may experience common selective forces that homogenize $\mathrm{C}$-terminal sequences within lineages. This hypothesis is intriguing given the recent evidence that MaSp1 and MaSp2 C-termini play a role in fiber formation $(S 7)$.

The phylogenetic distribution of deinopoid and araneoid spidroins involved in orb-web construction, particularly Flag and MaSp2, provides additional support for the monophyly of Orbiculariae and therefore, the single, ancient origin of orb-web architecture. An alternative explanation, that Flag and/or MaSp2 spidroins evolved convergently in these two lineages, would require thousands of additional nucleotide mutations to arrive at such similar protein sequences.

\section{References}

S1. J. E. Garb, C. Y. Hayashi, Proc. Natl. Acad. Sci. USA 102, 11379 (2005).

S2. J. Gatesy, C. Hayashi, D. Motriuk, J. Woods, R. Lewis, Science 291, 2603 (2001).

S3. P. L. Tai, G. Y. Hwang, I. M. Tso, Int. J. Biol. Macromol. 34, 295 (2004).

S4. D.L. Swofford, PAUP* version 4.0b10. Sinauer Associates, Sunderland, Mass. (2002).

S5. J.P. Huelsenbeck, F. Ronquist, Bioinformatics 17, 754 (2001).

S6. M. B. Hinman, R. V. Lewis, J. Biol. Chem. 267, 19320 (1992).

S7. A. Sponner et al., Biochem. Biophys. Res. Commun. 338, 897 (2005). 
A.

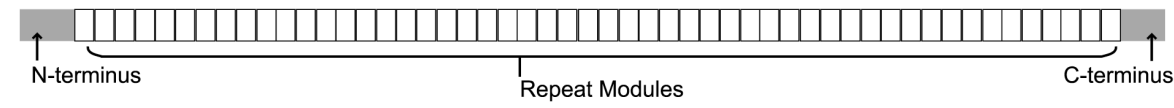

B. Silk Protein Repeat Consensus Sequence

cDNA Length

D.s. fibroin 1a

D.s. fibroin $1 \mathrm{~b}$

U.d. MaSp1

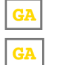

U.d. MaSp

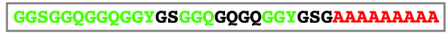

D.s. MaSp2a

D.s. MaSp2b

GXGPGXQGPGXOGPGGYGPGAAAAAAAA

GPGGYGGPGOOGPGOGOYGPGTGOOGOGPASAAAAAAA

GPGGYGGPGQQGPGQGQQOGPASAAAAAAAAAA

U.d. MiSp1

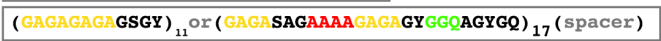

D.s. MiSp1

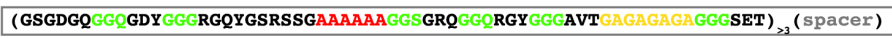

(spacer) (GPQGGGG)

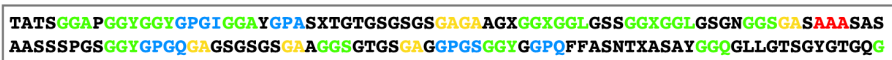
GASGTGS GGGGSGSGTGSNS $\triangle$ SSAAAAAAA

D.s. fibroin 2

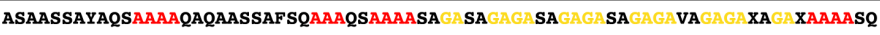

D.s. TuSp1 AAASSSASAVASAFAQSASYALASSSAFANAFASATSAGYLGSLAYQLGLTTAYNLGLSNAQAFANTLSQAVXG VGV SASAYASAISNAVGPYLYGLGLFNQANAASFASSFASAVSSAVASAS

AAASASSAASAQSAAAALAQSQSAASAF SQAASQAFSQAASQAGSQAASQAGSQAASQAGSQAASQAGSQAAS QAGSQAASQAXSQXAXXSGSGASSFTTTISRSSAGSQXGSQAGSQAGSQAGSQAGSQAGSQAGSQAGSQAGSQ AGSQAGSQAGSQAGSQAASQAGSQSASQASASSSASAFASASAFAQSASFALSSSSSFASAVSSASSXSALGT LGYQVGLQAAGSLGISNSQAFASSISQALTSVGVGASSSAYASAVSGVVAQYLSGTGVLTSANAQALASSFAN VFAASAASAS

U.d. TuSp1

STASSLGIQLGASLGAGFGASAGLSASTDISSSVEATSASTLSSSASSTSVVSSINAQLVPALAQTAVLNAAFS NINTQNAIRIAELLTQQVGRQYGLSGSDVATASSQIRSALYSVQQGSASSAYVSAIVGPLITALSSRGVVNASN SSQIASSLATAILQFTANVAPQFGISIPTSAVQSDLSTISQSLTAISSQTSSSVDSSTSAFGGISGPSGPSPYG POPSGPTFGPGPSLSGLTGFTATFASSFKSTLASSTOFOLIAOSNLDVQTRSSLISKVLINALSSLGISASVAS SIAASSSQSLLSVSAGASAADIATAIAASVATSLQSNGVLTASNVSQLSNQLASYVSSGLS

C.

PGQOGPGQG $Q Y G P G T G Q Q G Q$ GPGG $Q G P V G A A A A A A A N V S$ GGYGSQGAGQGGQQGSGQGGPAAAGPGGYGGPG QOGPEOGOYGSGAGOOGOGPGOOGPASAAAAAAAGPGRYGGPGOOGPGOGOYGPGTGOOGOGPASAAAAAAAG PGGYGGPGOOGPGOGRTGOOGOGLGGOOGPDSAAAAAAAAGPGGYGGPGOOGPGOGOYGPGTGOOGOGPGOO GPVGAAAAAAAAVSSGGYGSOGAGOGGOOGSGORGPAAAGPGGYSGPGOOGPGOGGOOGPASAAAAAAAAAGPG GYVGSGOOGPGOGRGTGOOGOGTGGOOGPASAASAAAGPGYYGPG G YGGPQD

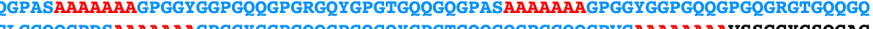

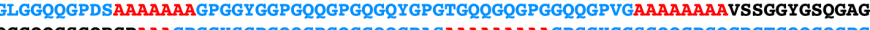
QGGQQGSGQRGPAAAGPGGYSGPGQOGPGQGGQOGPASAAAAAAAAAGPGGYGGSGQOGPGQGRGTGQOGQGPG GQQGPASAAAAAAAGPGGYGGPGQQGPGQGQYGPGTGQOGOGPASAAAAAAAGPGGYGGPGQGPGQGQYGPGT GQQGOGPGGQQPGGASAAAAAAAGPGGYGGPGQQGGGQQAPVQPRPYVPVTSGQIVTSDVSSTVSSAVSRMST PGSGSRISNAVSNILSSGVSSSSGLSNAISNISSS ISASNPGLSGCDVLVQVLLEVISALVHILGSASVGQVGS SPQNAQMVAANAVANAFS.

D. D.s. SGP-1
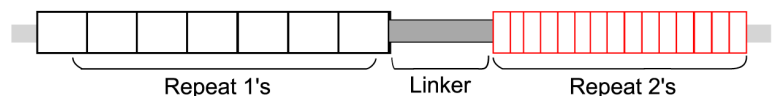

E. Repeat 1 NAAANSXGYGGY PYGYYRGGLSYSDASADASALSSSLGGLSSSXAAA

Linker

Repeat 2

GPAYYDYELLYP YYYDDYYVRGPAGGV SLSSAGAASTAVS PPGGAAAGSAAEASSLAMGSRYGRRYGLADSMASAEAS

NAAANANSIALGGRGGGLAAS

Fig. S1. Silk proteins expressed by deinopoid spiders have a diversity of modular architectures.

(A) Schematic of spidroin primary sequence showing that $~ 90 \%$ is composed of tandemly-iterated repeat modules. (B) Summary of 13 deinopoid spidroins listing the consensus of individual repeat modules (determined using methods in S2) in each protein and length of characterized cDNA. The four common amino acid motifs (S2) are indicated by color (yellow--GA, green--GGX, red--A ${ }_{n}$, and blue--GPX ${ }_{n}$ ). (C) Representative spidroin sequence (D.s. MaSp2a) with C-terminus in grey text. (D) Organization of Deinopis SGP-1 primary sequence. (E) Consensus of two SGP-1 repeats with intervening linker sequence highlighted in grey. 


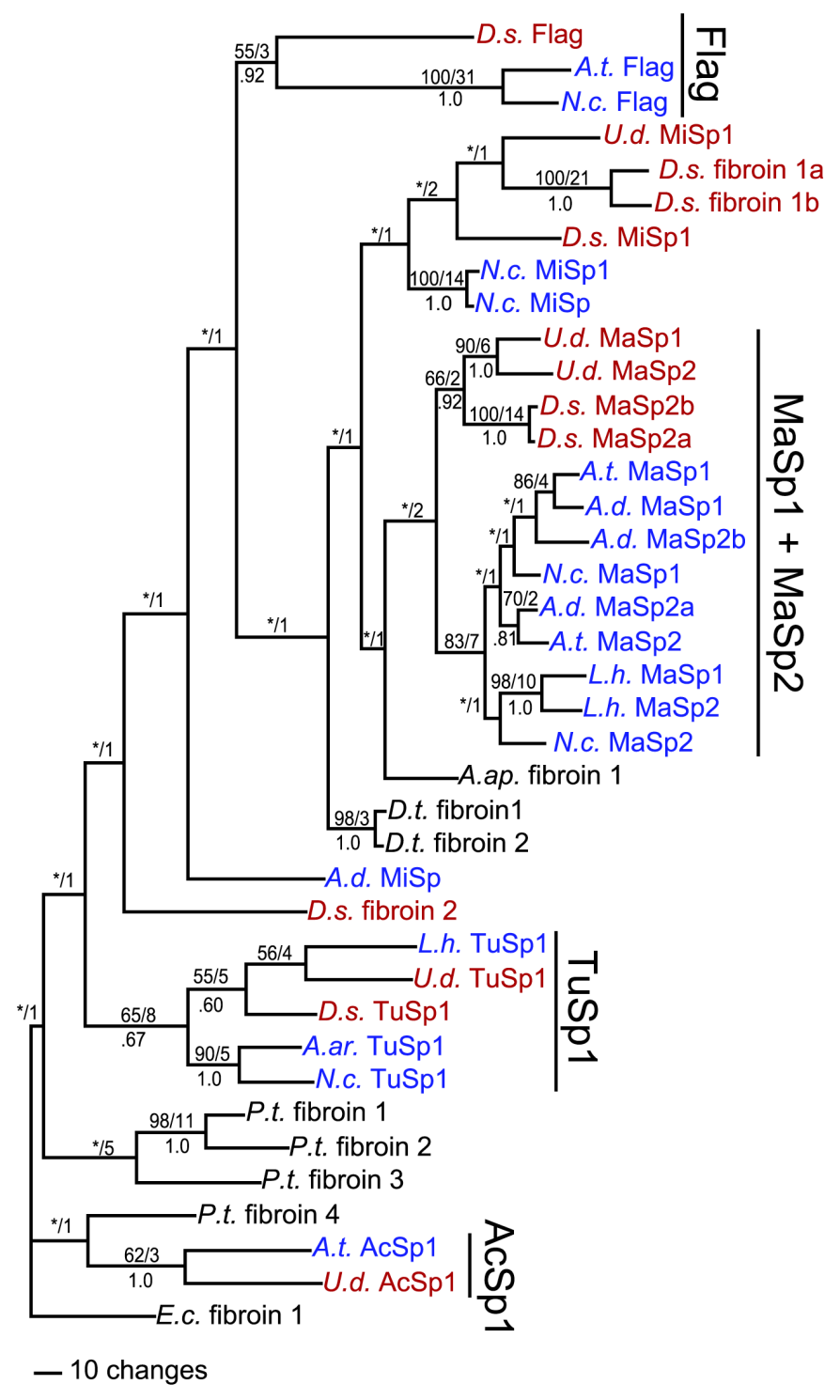

Fig. S2. Phylogenetic relationships of deinopoid and araneoid spidroins determined from C-terminal amino acid sequences. Phylogram of most parsimonious tree with numbers above internodes indicating bootstrap support/decay index, asterisks indicate nodes with $<50 \%$ bootstrap support and numbers below internodes are posterior probability values in majority rule consensus of post-burn-in Bayesian trees. Deinopoid spidroins in red text and araneoid spidroins in blue. Species abbreviations as follows: D.s. (Deinopis spinosa), U.d. (Uloborus diversus), A.t. (Argiope trifasciata), A.ar. (Argiope argentata), N.c. (Nephila clavipes), A.d. (Araneus diadematus), L.h. (Latrodectus hesperus), A.ap. (Agelenopsis aperta), D.t. (Dolomedes tenebrosus), P.t. (Plectreurys tristis), and E.c. (Euagrus chisoseus). 